\title{
Efecto de distintos tratamientos pregerminativos sobre la germinación de seis especies nativas de la región mediterránea de Chile
}

\author{
Effect of different pre-germination treatments on germination of six native \\ species from the mediterranean area of Chile
}

\author{
Ursula Doll ${ }^{*}$, Michelle Fredes V., Cecilia Soto V.
}

\begin{abstract}
RESUMEN
Muchas especies de la flora nativa del norte y centro de Chile se destacan por su capacidad de soportar condiciones edáficas extremas y presentar estrategias para sobrevivir en condiciones de escasez de agua, características especialmente favorables para poder ser utilizadas en la formación de cubiertas vegetales estabilizadores de taludes. En este contexto se eligieron seis especies nativas que se encuentran habitando frecuentemente taludes viales antiguos de la zona mediterránea de Chile, con el propósito de poder utilizarlas para revegetar taludes con la técnica de hidrosiembra. Se realizaron dos ensayos en cámara de germinación, con el objetivo de determinar sus requerimientos de germinación. Se evaluó el efecto de remojo en agua fría, remojo en agua caliente y estratificación fría como tratamientos pregerminativos, sobre la capacidad germinativa de las semillas. Las semillas de Melica violacea estratificadas germinaron a los cinco días y las semillas de Baccharis linearis remojadas en agua caliente mostraron al día siete su máxima energía germinativa, alcanzando una capacidad germinativa de $99 \%$ y $91 \%$, respectivamente. Ambas especies se perfilan como promisorias para ser usadas para la revegetación de taludes mediante la técnica de hidrosiembra.
\end{abstract}

Palabras clave: capacidad germinativa, Melica violacea, Baccharis linearis, Proustia cuneifolia, Haplopappus sp.

\begin{abstract}
Many species of the native flora from north and central Chile stands out in their capacity to withstand extreme edaphic conditions and also presents strategies to survive under water deficit conditions. These characteristics make them especially amenable to be used in vegetable covers to stabilize slopes. In this context, 6 native species which usually grow on old road slopes in the mediterranean area of Chile were chosen to be used to revegetate slopes using the hydroseeding technique. 2 trials were carried out in a germination chamber with the aim to determinate the seeds' germination requirements. The germination capacity was tested under pre-germination treatments as soaking in either cold or hot water, and cold stratification. Stratified seeds of Melica violacea germinated after 5 days, and seeds of Baccharis linearis soaked in hot water showed their maximum germination energy on day 7, reaching a germination capacity of $99 \%$ and $91 \%$ respectively. Both species seems to be promising for revegetating slopes with the hydroseeding technique.
\end{abstract}

Key words: germination capacity, Melica violacea, Baccharis linearis, Proustia cuneifolia, Haplopappus sp.

\section{Introducción}

La fuerte pendiente, baja fertilidad y escasa retención hídrica que caracterizan la mayoría de los taludes viales impiden el establecimiento exitoso de una cubierta vegetal mediante técnicas tradicionales de siembra o plantación. Razón por ello se desarrolló la técnica de hidrosiembra que es considerada como una de las herramientas más eficaces para controlar y prevenir la erosión y la sedimentación en terraplenes y cortes de caminos.
Esta técnica consiste en la proyección vía aspersión de una mezcla homogénea de agua, semillas, mulch, adherente y fertilizante sobre el terreno desnudo (Sheldon y Bradshaw, 1977).

Sin embargo, en condiciones climáticas mediterráneas y semiáridas el uso de mezclas comerciales de rápido crecimiento como iniciadoras de la revegetación mediante la técnica de hidrosiembra no siempre resulta exitoso (Matezanz et al., 2006). García-Palacios et al. (2010) sugieren utilizar mezclas de semillas autóctonas para promover la

\footnotetext{
$1 \quad$ Facultad de Ciencias Forestales, Universidad de Talca, Casilla 721-747, Talca, Chile.

* Autor por correspondencia: udoll@utalca.cl
} 
colonización natural de los taludes, ya que el uso de mezclas comerciales en terraplenes puede resultar en una supresión de la vegetación nativa. Tormo et al. (2008) encontraron que el estrés hídrico es el factor más influyente en el éxito de establecimiento y permanencia de las especies en terraplenes de clima mediterráneo. Por otro lado, Bochet et al. (2010) sostienen que la disponibilidad de micrositios es un factor limitante para la instalación de las especies, a su vez lograron los mejores resultados aumentando la rugosidad de la superficie del talud e hidrosembrando especies nativas seleccionadas.

Muchas especies de la flora chilena de la zona central y norte del país se destacan por su capacidad de adaptarse a condiciones edáficas extremas, habiendo desarrollado muchas de ellas estrategias para sobrevivir en condiciones de escasez de agua. Estas características son especialmente deseadas en especies que puedan ser utilizadas para formar cubiertas vegetales restauradoras (Rondon y Vidal, 2005). Sin embargo, existe escasa información acerca de condiciones y requerimientos de germinación de las distintas especies.

En este contexto se eligieron seis especies nativas que se encuentran habitando frecuentemente taludes viales antiguos de la zona mediterránea de Chile, con el propósito de poder utilizarlas para revegetar taludes con la técnica de hidrosiembra.

Melica violaceae Cav. de la familia de las Graminae, es una especie herbácea perenne con hábito cespitoso. Crece en suelos arenosos y pedregosos (Riedemann y Aldunate, 2003). Haplopappus scaposus J. Rémy, Haplopappus pulchellus (Bertero) DC., Baccharis linearis (Ruiz \& Pav.) Pers. y Proustia cuneifolia D. Don. pertenecen a la familia de las Compositae. Habitan suelos secos y pedregosos. Ambas especies del género Haplopappus son perennes, florecen en primavera y poseen hojas coriáceas y resinosas. H. scaposus, cuyas flores son de color naranja, forma varios tallos cortos en roseta, mientras que $H$. pulchellus posee un hábito más erecto y flores amarillas (Reiche, 1905; Navas, 1979). Baccharis y Proustia son arbustos de gran tamaño capaces de crecer en suelos degradados y pendientes pronunciadas (Riedemann y Aldunate, 2001). Florecen en verano, siendo el primero dioico y perennifolio, mientras que el segundo, cuyas ramas terminan en espinas, es caducifolio en invierno. Trevoa quinquenervia Gillies \& Hook. de la familia de las Rhamnaceae, es un arbusto capaz de vivir en suelos degradados y con fuerte pendiente. Florece abundantemente en primavera, posee espinas y su follaje es caducifolio en verano (Riedemann y Aldunate, 2001).

Las seis especies seleccionadas se caracterizan por su rusticidad y capacidad de vivir en ambientes secos, siendo el objetivo de este trabajo estudiar sus requerimientos de germinación.

\section{Material y Métodos}

Se realizaron 2 ensayos de germinación dentro de una cámara de germinación, que consiste en un recinto cerrado de $4,6 \mathrm{~m}^{2}$, dentro del cual un estante metálico sirve para la disposición de las bandejas de germinación. La temperatura e iluminación del recinto son regulables. En el primer ensayo realizado entre septiembre y octubre del 2006 se utilizaron semillas de Baccharis linearis, Proustia cuneifolia y Trevoa quinquenervia recolectadas entre abril y mayo del mismo año en los alrededores de Talca. El segundo ensayo se desarrolló entre enero y marzo de 2007, usando semillas de Melica violacea, Haplopappus scaposus y Haplopappus pulchellus recolectadas también en los alrededores de Talca durante el verano 2005-2006.

Las semillas fueron limpiadas y almacenadas dentro de bolsas de papel en ambiente seco y a temperatura ambiente hasta ser sometidas a los diferentes tratamientos pregerminativos. El tratamiento remojo en agua fría consistió en sumergir las semillas previo al ensayo durante 24 horas en agua a temperatura ambiente, mientras que para el remojo en agua caliente las semillas fueron sumergidas en agua a una temperatura de $70{ }^{\circ} \mathrm{C}$, que se dejó enfriar posteriormente por 24 horas. La estratificación consistió en colocar las semillas previamente embebidas durante 60 días a temperatura baja $\left(4-6{ }^{\circ} \mathrm{C}\right)$. El tratamiento testigo fue similar al remojo en agua fría pero sin aplicación de fungicida.

Por flotación fueron separadas en cada caso las semillas no viables. Las semillas viables ya tratadas fueron colocadas dentro de cajas Petri forradas en su interior con papel absorbente humedecido con solución fungicida $\left(1 \mathrm{~g} \times \mathrm{L}^{-1}\right.$ Captan, 13,04 g i.a. en $100 \mathrm{~g}$ ). En el caso del tratamiento testigo el papel absorbente solo fue humedecido con agua. Las cajas Petri fueron selladas con filme plástico transparente para evitar la contaminación. Posteriormente se introdujeron en la cámara de germinación a $20-25^{\circ} \mathrm{C}$ e iluminación continua. 
En el primer ensayo se colocaron 25 semillas por caja de Petri y se contó con cuatro repeticiones por tratamiento. En el segundo ensayo se colocaron 30 semillas por caja, contándose con cinco repeticiones por tratamiento. Periódicamente se revisaron las cajas, contabilizando las semillas germinadas en cada caso y se volvía a humedecer el papel absorbente de ser necesario.

Los porcentajes de germinación acumulados a los 27 días en el primer ensayo y a los 47 días en el segundo ensayo, fueron sometidos a un análisis de varianza independiente para cada especie. Para su evaluación estos valores expresados en porcentaje fueron modificados mediante la transformación angular.

Para cada tratamiento se calculó además la capacidad germinativa (porcentaje de germinación acumulado al final del ensayo), el valor máximo (máximo cuociente logrado entre el porcentaje de germinación acumulado en un período determinado y el número de días que comprende ese período), la energía germinativa (porcentaje de germinación acumulado hasta el día en que se alcanza el valor máximo) y el período de energía (número de días que tarda en ocurrir el valor máximo) (Cabello et al. 2001-2002).

\section{Resultados y Discusión}

Melica violácea destacó por su alta capacidad germinativa lograda en todos los tratamientos pregerminativos, con un promedio superior al $90 \%$ de germinación (Tabla 1). Casi la totalidad de las semillas de esta especie sometidas a estratificación germinaron. Las sometidas a remojo en agua caliente disminuyeron significativamente su capacidad germinativa, alcanzando solo cerca de un $80 \%$ de germinación. Por otro lado pudo comprobarse un efecto inhibidor del fungicida, ya que las semillas sometidas a agua fría lograron un porcentaje mayor de germinación cuando no se les aplicó fungicida (97\% contra $87 \%$, Tabla 1). Sin embargo, fue necesario interrumpir el tratamiento testigo antes de la finalización del ensayo, al igual que en el caso de las dos especies de Haplopappus, debido a la gran contaminación fúngica que presentaban las placas respectivas (Figura 1).

También destacó Baccharis linearis cuyas semillas sometidas a agua caliente germinaron en un $91 \%$. Las semillas estratificadas de esta especie y las sometidas a agua fría lograron un porcentaje de germinación significativamente menor (46\% y $26 \%$ respectivamente, Tabla 1). No se realizó el tratamiento testigo en Baccharis por una baja disponibilidad de semillas.

Las semillas de Proustia cuneifolia estratificadas y remojadas en agua fría y agua caliente también mostraron una buena capacidad germinativa, alcanzando un porcentaje de germinación promedio de $76,3 \%$ para los tres tratamientos (Tabla 1). El resultado obtenido en las semillas testigo indica que es indispensable la aplicación de un fungicida, ya que la aparición de hongos deprimió significativamente la germinación de las semillas (Figura 1).

Tanto Haplopappus scaposus como Haplopappus pulchellus mejoraron significativamente su capacidad germinativa al someter sus semillas a estratificación, logrando $61 \%$ y $36 \%$ de germinación respectivamente (Tabla 1). Sin embargo, en el caso de H. pulchellus menos de la mitad de las semillas alcanzó a germinar. Ningún otro tratamiento en ambas especies logró más de un $5 \%$ de germinación (Figura 1). Vogel et. al. (2008) encontraron diferencias en la capacidad de germinación de diferentes especies del género

Tabla 1. Capacidad germinativa [\%] de semillas de seis especies nativas sometidas a distintos tratamientos pregerminativos.

\begin{tabular}{lrrrr}
\hline \multirow{2}{*}{ Tratamiento $\rightarrow$} & \multicolumn{4}{c}{ Porcentaje de germinación } \\
\cline { 2 - 5 } & Agua fría & Agua caliente & Estratificación & Testigo \\
\hline Trevoa quinquenervia & $24 \pm 3,27$ & $9 \pm 8,25$ & $3 \pm 3,83$ & $10 \pm 4,00$ \\
Proustia cuneifolia & $76 \pm 5,66 \mathrm{a}$ & $73 \pm 2,00 \mathrm{a}$ & $80 \pm 10,33 \mathrm{a}$ & $45 \pm 10,00 \mathrm{~b}$ \\
Baccharis linearis & $26 \pm 36,59 \mathrm{~b}$ & $91 \pm 7,57 \mathrm{a}$ & $46 \pm 14,79 \mathrm{~b}$ & \\
Melica violacea & $87 \pm 3,33 \mathrm{c}$ & $79 \pm 5,96 \mathrm{c}$ & $99 \pm 1,49 \mathrm{a}$ & $97 \pm 2,36 \mathrm{~b}$ \\
Haplopappus pulchellus & $5 \pm 3,80 \mathrm{~b}$ & $5 \pm 5,58 \mathrm{~b}$ & $36 \pm 10,11 \mathrm{a}$ & $3 \pm 4,71 \mathrm{~b}$ \\
Haplopappus scaposus & $4 \pm 1,49 \mathrm{~b}$ & $2 \pm 2,98 \mathrm{bc}$ & $61 \pm 8,69 \mathrm{a}$ & $1 \pm 1,49 \mathrm{c}$ \\
\hline
\end{tabular}

Los valores seguidos de distintas letras en la fila presentan diferencias estadísticamente significativas, según prueba de Rangos Múltiples de Duncan, con $P \leq 0,05$. 


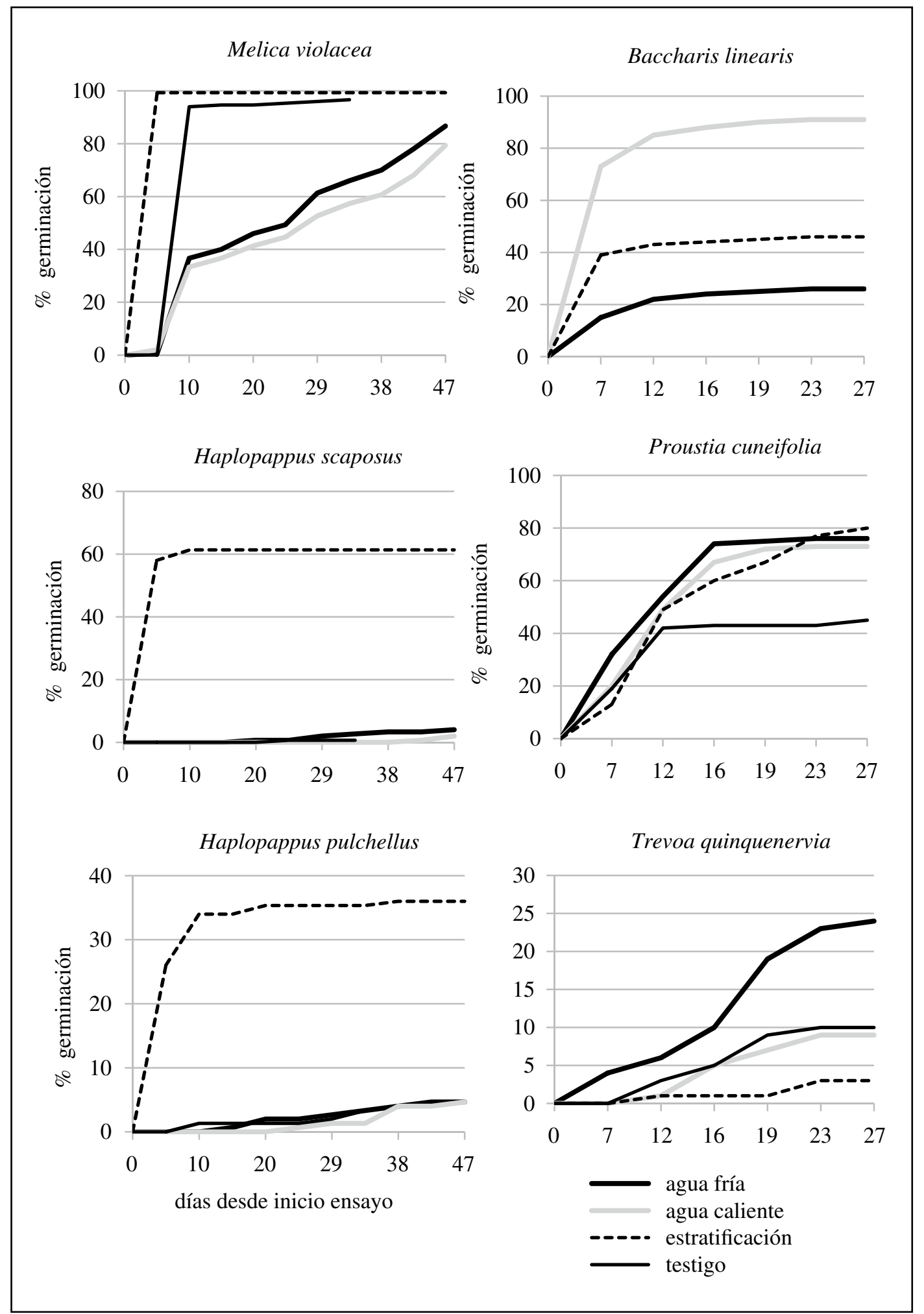

Figura 1. Germinación acumulada para semillas de seis especies nativas sometidas a distintos tratamientos pregerminativos. 
Tabla 2. Capacidad germinativa [\%], valor máximo [\%], energía germinativa [\%] y período de energía [días] de semillas de seis especies nativas, para los tratamientos pregerminativos que alcanzaron la mayor capacidad germinativa respectivamente.

\begin{tabular}{|c|c|c|c|c|c|c|}
\hline & $\begin{array}{l}\text { Melica } \\
\text { violacea }\end{array}$ & \multicolumn{2}{|c|}{$\begin{array}{c}\text { Haplopappus pulchellus } \\
\text { scaposus }\end{array}$} & $\begin{array}{c}\text { Proustia } \\
\text { cuneifolia }\end{array}$ & $\begin{array}{c}\text { Baccharis } \\
\text { linearis }\end{array}$ & $\begin{array}{c}\text { Trevoa } \\
\text { quinquenervia }\end{array}$ \\
\hline Tratamiento $\rightarrow$ & \multicolumn{4}{|c|}{ estratificación } & a. caliente & agua fría \\
\hline capacidad germinativa [\%] & 99,3 & 36 & 61,3 & 80 & 91 & 24 \\
\hline valor máximo [\%] & 19,8 & 5,2 & 6,1 & 4,1 & 10,4 & 0,8 \\
\hline energía germinativa [\%] & 99,3 & 26 & 61,3 & 49 & 73 & 24 \\
\hline período de energía [días] & 5 & 5 & 10 & 12 & 7 & 27 \\
\hline
\end{tabular}

Haplopappus. Reportaron porcentajes de germinación superiores al $80 \%$ con remojo en agua y diferente respuesta a la germinación en luz y oscuridad para $H$. baylahuen, $H$. multifolius y $H$. remyanus. Por otro lado $H$. taeda requirió de un tratamiento de escarificación para mejorar su capacidad germinativa.

Trevoa quinquenervia mostró una baja capacidad germinativa con los tratamientos ensayados, alcanzando solo un $24 \%$ de germinación al ser las semillas remojadas en agua fría (Tabla 1). En el caso de esta especie no se cumplieron los supuestos de homogeneidad de la varianza para su análisis. Los porcentajes de germinación logrados con los distintos tratamientos pregerminativos se mantuvieron dentro de los rangos que reportaron Cabello et. al. (2001-2002) para semillas de esta especie sometidas a remojo en agua. Los mismos autores indican que las semillas presentan latencia exógena física, que debe ser interrumpida con remojo en $\mathrm{H}_{2} \mathrm{SO}_{4}$ concentrado.

Silva et al. 2011 reportaron que tanto la germinación como el vigor de las plántulas formadas por semillas de Daucus carota L. son afectadas por condiciones de estrés hídrico. Laynez-Garsaball et al. (2007) determinaron que bajos potenciales agua del sustrato afectan el crecimiento de plántulas de Zea mays L., independiente del tamaño de la semilla. Por otro lado Bochet et al. (2010) encontraron que las especies exitosas para colonizar taludes en ambiente semiárido mediterráneo presentan tasas y velocidades de germinación más altas que las especies no exitosas, en condiciones experimentales de baja disponibilidad hídrica. A su vez reportaron que la mayor parte del tiempo los estratos superiores de los taludes permanecen secos, además de secarse rápidamente después de eventos de precipitación.
Por lo tanto, son capaces de colonizar taludes en ese ambiente, las especies cuyas semillas germinan rápidamente con bajos contenidos de agua en el sustrato.

Bajo las condiciones ensayadas Melica, Baccharis, Proustia y las semillas estratificadas de Haplopappus comenzaron a germinar antes de los siete días, mientras que Trevoa a los 12 días (Figura 1). Las semillas estratificadas de Melica incluso ya el día cinco alcanzaron su máxima capacidad germinativa (Tabla 2). También las semillas estratificadas de H. pulchellus mostraron el día cinco su máxima energía germinativa, pero alcanzando una capacidad germinativa de solo $36 \%$. Las semillas de Baccharis remojadas en agua caliente alcanzaron en siete días su máxima energía germinativa, mientras que las semillas estratificadas de H. scaposus y Proustia tardaron 10 y 12 días respectivamente (Tabla 2). A excepción de Trevoa, todas las especies son capaces de germinar en períodos relativamente cortos con los tratamientos pregerminativos adecuados, destacándose especialmente Melica y Baccharis que además presentaron las capacidades germinativas más altas.

\section{Conclusiones}

En base a los resultados obtenidos frente a los tratamientos pregerminativos ensayados, Melica violacea y Baccharis linearis se perfilan como dos especies promisorias para ser utilizadas en la revegetación de taludes mediante la técnica de hidrosiembra. Ambas especies germinaron dentro de los siete días de ser remojadas las semillas de Melica solo con agua fría (tratamiento testigo) y las semillas de Baccharis con agua caliente, alcanzando porcentajes de germinación superiores al $90 \%$. 


\section{Literatura Citada}

Bochet, E.; García-Fayos, P.; Tormo, J.

2010. How can we control erosion of roadslopes in semiarid Mediterranean areas? Soil improvement and native plant establishment. Land Degradation \& Development, 21: 110-121.

Cabello, A.; Sandoval, A.; Carú, M.

2001-2002. Efecto de los tratamientos pregerminativos y de las temperaturas de cultivo sobre la germinación de semillas de Talguenea quinquenervia (talguén). Ciencias Forestales, 16 (1-2): 11-18.

García-Palacios, P.; Soliveres, S.; Maestre, F.; Escudero, A.; Castillo-Monroy, A.; Valladares, F.

2010. Dominant plant species modulate responses to hydroseeding, irrigation and fertilization during the restoration of semiarid motorway slopes. Ecological Engineering, 36 (10): 1290-1298.

Laynez-Garsaball, J.; Méndez, J.; Mayz-Figueroa, J.

2007. Crecimiento de plántulas a partir de tres tamaños de semilla de dos cultivares de maíz (Zea mays L.), sembrados en arena y regados con tres soluciones osmáticas de sacarosa. Idesia, 25 (1):21-36.

Matesanz, S.; Valladares, F.; Tena, D.; Costa-Tenorio, M.; Bote, D. 2006. Early Dynamics of Plant Communities on Revegetated Motorway Slopes from Southern Spain: Is Hydroseeding Always Needed? Restoration Ecology, 14 (2): 297-307.

Navas, L.

1979. Flora de la Cuenca de Santiago de Chile. Tomo III. Editorial Universitaria, Santiago, Chile, pp. 188-189.

Reiche, C.

1905. Flora de Chile. Tomo Cuarto. Imprenta Cervantes, Santiago, Chile. disponible en http://flora.huh.harvard.edu/ FloraData/060/PDF/V04/Volume4-Part1_ Haplopappus_ Name_Prob.pdf
Riedemann, P.; Aldunate, G.

2001. Flora nativa de valor ornamental, identificación y propagación: Chile Zona Centro. Editorial Andrés Bello, Santiago, Chile, 566 p.

Riedemann, P.; Aldunate, G.

2003. Flora nativa de valor ornamental, identificación y propagación: Chile Zona Sur. Editorial Andrés Bello, Santiago, Chile, 516 p.

Rondón, J.; Vidal, R.

2005. Establecimiento de la cubierta vegetal en áreas degradadas (principios y métodos). Revista Forestal Latinoamericana, 20 (38): 63-82.

Sheldon, J.; Bradshaw, A.

1977. The development of hydraulic seeding technique for instable sand slopes. Journal of Applied Ecology 14 (3): 905-918.

Silva, M.; Medeiros, A.; Dias, D.; Alvarenga, E.; Coelho, F.;

Braun, $\mathrm{H}$.

2011. Efeito do estresse hídrico e térmico no germinaçâo e no vigor de sementes de cenoura. Idesia, 29 (3): 39-44.

Tormo, J.; García-Fayos, P.; Bochet, E.

2008. Relative importance of plant traits and ecological filters in road embankment revegetation under semiarid Mediterranean conditions. Ecological Engineering, 33: 258-264.

Vogel H.; Razmilic, I.; San Martín, J.; Doll, U.; González, B. 2008. Plantas medicinales chilenas. Experiencias de domesticación y cultivo de Boldo, Matico, Bailahuén, Canelo, Peumo y Maqui, $2^{\text {a }}$ edición. Editorial de la Universidad de Talca, Talca, Chile, 194 p. 